\title{
Deciphering the genetic basis of common diseases by integrated functional annotation of common and rare variants
}

\author{
Olivier Harismendy ${ }^{1,2^{*}}$, Gaurav Bhatia ${ }^{3}$, Nazli G Rahim ${ }^{4}$, Vikas Bansal ${ }^{4}$, Masakazu Nakano ${ }^{4}$, Michael Scott ${ }^{5}$, \\ Xiaoyun Wang ${ }^{1,2}$, Colette Dib ${ }^{6}$, Edouard Turlotte ${ }^{6}$, Nathaniel Heintzman $^{7}$, Sarah S Murray ${ }^{4}$, Jean-Francois Deleuze ${ }^{6}$, \\ Jack C Sipe ${ }^{5}$, Bing Ren ${ }^{7}$, Vineet Bafna ${ }^{3}$, Eric J Topol ${ }^{4}$, Kelly A Frazer ${ }^{1,2}$
}

From Beyond the Genome: The true gene count, human evolution and disease genomics Boston, MA, USA. 11-13 October 2010

Genome-wide associations studies (GWAS) have identified $>400$ loci associated with common human diseases. Despite the extraordinary achievement that these results represent, several questions remain unanswered: (i) What is the contribution of rare variants to common diseases not addressed by GWAS? (ii) What are the functional variants underlying the GWAS associations?

To answer these questions, we first explored the role of common and rare variants in morbid obesity (BMI $>40)$ by identifying all the variants in the intervals of two endocannabinoid metabolic genes (MGLL and $F A A H)$ using targeted sequencing. We used two different statistical approaches (single marker tests and collapsed markers tests) to identify variants in four intervals associated with high BMI. The majority of the associated variants are located in promoter sequences or in predicted transcriptional enhancers, suggesting their regulatory role. Additionally, we demonstrate that the set of rare variants in the $F A A H$ promoter associated with BMI are also associated with an increased level of anandamide, a FAAH substrate, further suggesting a functional role in obesity. In a second study, we examined one of the most compelling GWAS that had identified SNPs in the 9p21 gene desert associated with coronary artery disease (CAD) and Type 2 diabetes (T2D). We identified 33 enhancers in 9p21; the interval is the second most dense gene-desert for predicted enhancers and is six times denser than the whole genome. The activity of three of these enhancers is affected by regulatory variants that show allelic differences in
DNA-protein affinity and are strongly associated with either CAD or T2D. Some of these variants are predicted to disrupt the transcription-binding site relevant to CAD biology. Our study shows that the 9p21 gene desert is significantly enriched in enhancer elements and suggests the associations with CAD and T2D likely result from the cumulative contributions of multiple regulatory variants.

Taken together, our studies show that a comprehensive approach of human genetics that integrates rare variants and functional knowledge, such as regulatory elements or metabolic and cell-specific effects, can leverage the findings of GWAS and lead to a deeper functional understanding of the role of genetic variation in human diseases.

\section{Author details \\ ${ }^{1}$ Moores UCSD Cancer Center, USA. ${ }^{2}$ Department of Pediatrics, University of California Dan Diego, USA. ${ }^{3}$ Department of Computer Sciences, University of California San Diego, USA. ${ }^{4}$ Scripps Translational Institute, USA. ${ }^{5}$ Department of Molecular and Experimental Medicine, The Scripps Research Institute, USA. ${ }^{6}$ Sanofi-Aventis Evry Genetics Center, USA. ${ }^{7}$ Ludwig Institute, University of California San Diego, CA 92092, USA.}

Published: 11 October 2010

doi:10.1186/gb-2010-11-S1-18

Cite this article as: Harismendy et al:: Deciphering the genetic basis of common diseases by integrated functional annotation of common and rare variants. Genome Biology 2010 11(Suppl 1):18.

${ }^{1}$ Moores UCSD Cancer Center, USA

Full list of author information is available at the end of the article 